\title{
No Effect of Inactivated SARS-CoV-2 Vaccination on in vitro Fertilization Outcomes: A Propensity Score-Matched Study
}

\author{
Jialyu Huang',*, Leizhen Xia',*, Jiaying Lin ${ }^{2, *}$, Bangdong Liu ${ }^{3, *}$, Yan Zhao', Cailin Xin ', Xiaoyan $\mathrm{Ai}^{4}$, \\ Wenting $\mathrm{Cao}^{5}$, Xiaocui Zhang ${ }^{6}$, Lifeng Tian', Qiongfang Wu' \\ 'Center for Reproductive Medicine, Jiangxi Maternal and Child Health Hospital, Nanchang, People's Republic of China; ${ }^{2}$ Department of Assisted \\ Reproduction, Shanghai Ninth People's Hospital, Shanghai, People's Republic of China; ${ }^{3}$ Department of Laboratory Medicine, Huanan Hospital of \\ Shenzhen University, Shenzhen, People's Republic of China; ${ }^{4}$ Department of Gynecology, Jiangxi Maternal and Child Health Hospital, Nanchang, \\ People's Republic of China; ${ }^{5}$ Department of Otorhinolaryngology, The First Affiliated Hospital of Nanchang University, Nanchang, People's Republic of \\ China; ${ }^{6}$ Center for Reproductive Medicine, Shangrao Maternal and Child Health Hospital, Shangrao, People's Republic of China
}

*These authors contributed equally to this work

Correspondence: Lifeng Tian; Qiongfang Wu, Center for Reproductive Medicine, Jiangxi Maternal and Child Health Hospital, Nanchang, People's Republic of China, Email 64554076@qq.com; wuqfivf@I26.com

Purpose: To investigate the impact of inactivated SARS-CoV-2 vaccination on in vitro fertilization (IVF) outcomes.

Patients and Methods: This retrospective cohort study included 2185 patients undergoing fresh IVF cycles from June 1 st to September 13th 2021 in a single university-affiliated hospital. Vaccine administration information was collected and ascertained via immunization records. Patients with two dosages of inactivated SARS-CoV-2 vaccines (Sinopharm or Sinovac) were categorized into the vaccinated group $(n=150)$, while those unvaccinated were classified as control $(n=2035)$. Propensity score matching was performed to balance the baseline characteristics (14 covariates) between the two groups at a ratio of 1:4. The main outcome measures were the number of oocytes retrieved, good-quality embryo rate and clinical pregnancy rate.

Results: There were 146 women in the vaccinated group and 584 in the control group after matching. The number of oocytes retrieved $(9.9 \pm 7.1$ vs $9.9 \pm 6.7 ; P=0.893)$, good-quality embryo rate $(33.5 \pm 29.8 \%$ vs $29.9 \pm 28.6 \% ; P=0.184)$ and clinical pregnancy rate $(59.1 \%$ vs $63.6 \% ; P=0.507)$ were all similar between the two groups. In addition, no significant differences were observed regarding other cycle characteristics, laboratory parameters and pregnancy outcomes. The results were also comparable when vaccinated patients were subdivided into three categories based on the time interval from complete vaccination to cycle initiation: $\leq 1$ month, $>1-2$ months, and $>2$ months.

Conclusion: Our study provided the first-time evidence that inactivated SARS-CoV-2 vaccination in females did not result in any measurable detrimental effects on IVF treatment. Owing to the present limitations, further prospective studies with larger cohort size and longer follow-up are warranted to validate our conclusion.

Keywords: COVID-19, SARS-CoV-2, vaccination, in vitro fertilization

\section{Introduction}

The coronavirus disease 2019 (COVID-19) outbreak caused by the severe acute respiratory syndrome coronavirus 2 (SARS-CoV-2) infection has become a globally serious pandemic. By the end of September 2021, documented cases of COVID-19 have surpassed 233 million worldwide, with nearly five million deaths attributed to it. ${ }^{1}$ Compared with nonpregnant reproductive-aged women, pregnant women with COVID-19 also demonstrate increased risks of intensive care unit admission, need for mechanical ventilation, maternal death, stillbirth and other adverse outcomes. ${ }^{2-4}$

Mass vaccination presents a key strategy to mitigate illness and establish herd immunity for controlling the pandemic. As of September 28, 2021, a total of 6.1 billion anti-SARS-CoV-2 vaccine doses were administered globally and more 
than 2.2 billion in China. ${ }^{1,5}$ Antiviral vaccines can be broadly categorized into four types, including inactivated virus vaccines, viral-vector vaccines, nucleic acid vaccines and protein-based vaccines. ${ }^{6}$ Among them, inactivated vaccines are predominantly used in China for the advantages of relatively mature production technology and easy storage at $2^{\circ} \mathrm{C}$ to $8^{\circ} \mathrm{C}$ for years. ${ }^{7}$ Based on a randomized clinical trial involving 40,382 participants, the efficacy of inactivated vaccines was shown to be over $70 \%$ for the prevention of laboratory-confirmed symptomatic COVID-19. ${ }^{7}$

Following vaccination, commonly reported adverse effects include injection site events (eg, pain, redness, swelling) and systemic effects (eg, fatigue, headache, muscle or joint pain), with rare serious adverse events. ${ }^{7-9}$ Despite the reassuring safety profile, the vaccination campaign is accompanied by concerns regarding the possible detrimental immunity-related effects on female fertility. ${ }^{10,11}$ These unsubstantiated claims, spread through social media, have led to public apprehension and further resulted in vaccination reluctance and pregnancy plan postponement for many women. ${ }^{12,13}$ To date, only two Israeli studies have focused on the potential correlation between messenger ribonucleic acid (mRNA) COVID-19 vaccine and in vitro fertilization (IVF) outcomes. ${ }^{14,15}$ Similar results were found in ovarian response and embryological characteristics between vaccinated and control groups, and one study further reported an acceptable pregnancy rate of $30 \%$ in vaccinated women. ${ }^{15}$ The authors thus concluded that mRNA COVID-19 vaccine did not influence patients' performance during IVF treatment. Nonetheless, both studies are of small sample size $(<50)$, and there is still a lack of real-world data on the follow-up of inactivated vaccine administration among infertile patients.

The aim of the present study was to investigate comprehensively the effect of female inactivated SARS-CoV-2 vaccination on cycle characteristics, laboratory parameters and pregnancy outcomes during IVF treatment.

\section{Materials and Methods Study Design and Population}

This was a retrospective cohort study performed at the Center for Reproductive Medicine, Jiangxi Maternal and Child Health Hospital (JMCHH) affiliated with Nanchang University School of Medicine. The study protocol was approved by the Ethics Committee of JMCHH (No. 2021-02) and was conducted in accordance with the Declaration of Helsinki. Informed contents were obtained from patients for data collection with scientific use.

All women who underwent fresh IVF cycles between June 1st and September 13th 2021 were screened for eligibility and followed up to October 18th 2021. Before the start of treatment, patients were questioned about their past SARSCoV-2 infection and vaccination status. Vaccine administration information, including vaccine type, dose, date, manufacturer and batch number, were ascertained via immunization records, which could be easily accessed through Alipay, WeChat, GanFuTong and many other mobile apps in China. Patients were categorized into the vaccinated group if they had received two dosages of inactivated SARS-CoV-2 vaccines (Sinopharm or Sinovac) with a break of at least three weeks between each dose, while those unvaccinated were classified as control. The exclusion criteria were as follows: (i) a prior history of SARS-CoV-2 infection; (ii) incomplete vaccination with only the first dose finished; (iii) use of other types of SARS-CoV-2 vaccines, namely recombinant adenovirus type-5 vectored vaccine (CanSino) and recombinant tandem-repeat dimeric receptor-binding domain-based protein subunit vaccine (ZF2001) in China; ${ }^{16,17}$ (iv) oocyte donation or preimplantation genetic testing cycles; and (v) lost to follow-up or important data missing in the electronic medical system. Routinely, chest X-ray film was performed for all patients to screen for any pulmonary lesions before cycle initiation, and negative polymerase chain reaction test for SARS-CoV-2 RNA detection was required 48 hours before oocyte retrieval. The flowchart of the study is depicted in Figure 1.

\section{IVF Protocol}

Controlled ovarian stimulation was carried out in a depot gonadotropin-releasing hormone $(\mathrm{GnRH})$ agonist long protocol, a flexible $\mathrm{GnRH}$ antagonist protocol or other protocols including mild stimulation and progestin-primed ovarian stimulation. The starting dose of gonadotropin was determined by patients' age, body mass index (BMI) and ovarian reserve, and subsequently adjusted according to ovarian response as monitored by transvaginal ultrasound examination and serum sex hormone measurement. Once the dominant follicle reached a diameter of $20 \mathrm{~mm}$ or two leading follicles 


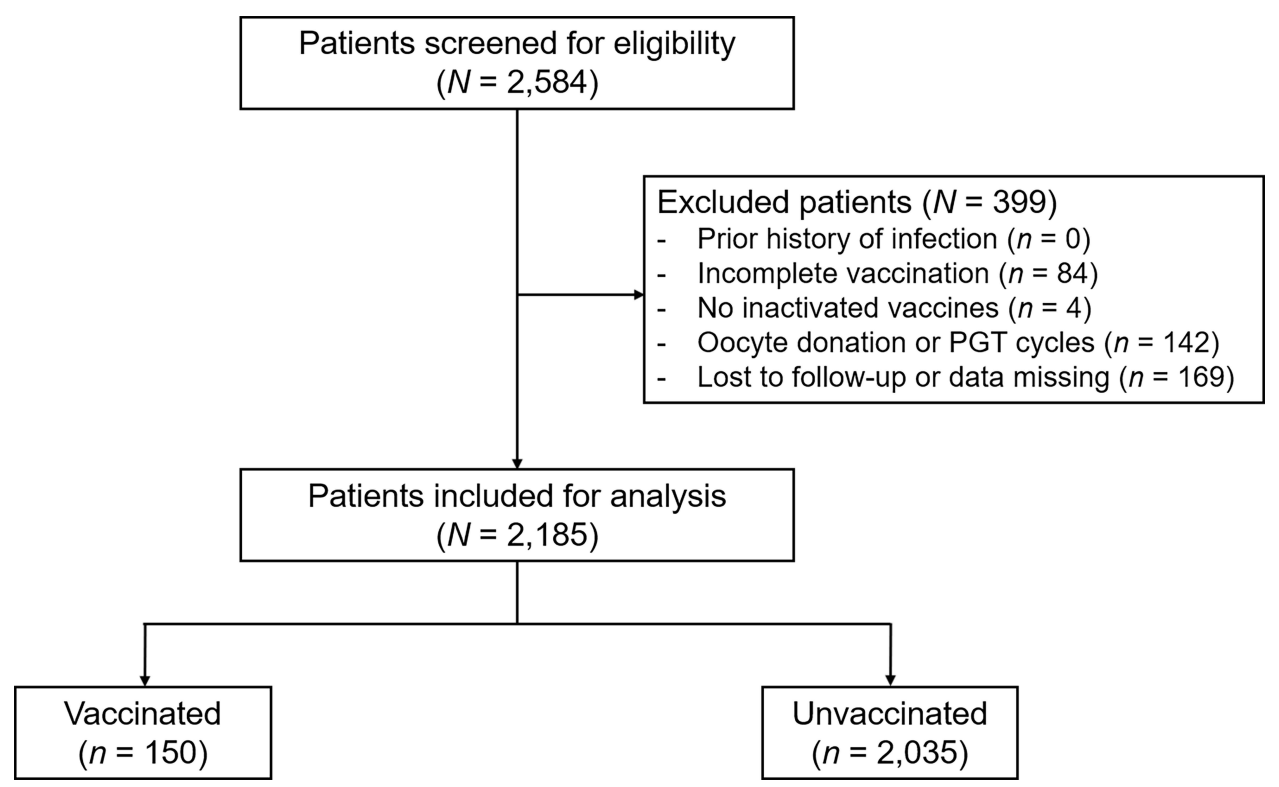

Figure I The flowchart of the study. Abbreviation: PGT, preimplantation genetic testing.

were over $18 \mathrm{~mm}, 250 \mu \mathrm{g}$ recombinant human chorionic gonadotropin (hCG; Ovidrel, Merck Serono, Switzerland) was injected to trigger ovulation.

Follicular aspiration was scheduled at 36-38 hours after triggering. The retrieved oocytes were inseminated by conventional IVF and/or intracytoplasmic sperm injection (ICSI) based on semen quality. Pronuclei (PN) evaluation was performed at 16-18 hours after fertilization. In a sequential manner, the zygotes were cultured to day 3 cleavage-stage embryos in G1-plus medium (Vitrolife, Sweden), and then transferred to the G2-plus medium for blastocyst culture until day 5 or 6 . According to the Cummins's morphological criteria, ${ }^{18}$ good-quality embryos on day 3 were defined as those with $7-10$ cells, $\leq 15 \%$ fragmentation, even blastomeres, and absence of multinucleation and vacuoles. The Gardner and Schoolcraft system was used to grade blastocysts, ${ }^{19}$ among which those with an expansion score $\geq 3$ and an inner cell mass or trophectoderm score $\geq \mathrm{B}$ were defined as available. The surplus embryos were cryopreserved using vitrification.

The embryo transfer strategy is mainly based on embryo quality/quantity on day 3 as well as the risk of ovarian hyperstimulation syndrome (OHSS). In detail, patients with over three good-quality embryos on day 3 would be suggested for blastocyst transfer on day 5, while those with fewer good-quality cleavage-stage embryos are advised to transfer immediately due to the likelihood of blastocyst formation failure and no viable embryos as a result. On the other hand, cleavage-stage embryo transfer would be allowed if the largest diameter of ovary is $<7 \mathrm{~cm}$ with serum estradiol level below $3000 \mathrm{pg} / \mathrm{mL}$ on day 3 after oocyte retrieval, while blastocyst transfer or freeze-all strategy could be performed for patients with higher chance of OHSS after re-examination on day 5. For patients undergoing fresh embryo transfer, endometrial transformation was induced with injected progesterone (60 mg/d; Xianju Pharma, China) from the day of oocyte retrieval. With the guidance of transabdominal ultrasound, up to 2 embryos were transferred 3 or 5 days later depending on the embryo developmental stage. Luteal phase support was provided using both vaginal progesterone gel $(90 \mathrm{mg} / \mathrm{d}$; Crinone, Merck Serono, Switzerland) and oral dydrogesterone (20 mg/d; Duphaston, Abbott Biologicals, USA), and continued until 10 weeks of gestation when a pregnancy was achieved.

\section{Outcome Measures and Definitions}

For laboratory outcomes, the oocyte retrieval rate was calculated as the ratio of the number of retrieved oocytes to the number of $\geq 14 \mathrm{~mm}$ follicles on trigger day. The mature oocyte rate was the ratio of the number of metaphase II (MII) oocytes to the number of oocytes retrieved. The normal fertilization rate was the ratio of the number of $2 \mathrm{PN}$ oocytes to the number of MII oocytes. The cleavage rate was the ratio of the number of day 3 cleavage-stage embryos developed 
from 2PN oocytes to the number of 2PN oocytes. The good-quality embryo rate was the ratio of the number of goodquality embryos on day 3 to the number of cleavage-stage embryos. The blastocyst formation rate was the ratio of the number of blastocysts to the number of day 3 embryos for extended culture. The available blastocyst rate was the ratio of the number of available blastocysts to the number of blastocysts formed on day $5 / 6$.

For pregnancy outcomes, biochemical pregnancy was defined as serum $\beta$-hCG concentration of $\geq 5 \mathrm{mIU} / \mathrm{mL}$ at 12 days following transfer. Clinical pregnancy was defined as the detection of at least one intrauterine gestational sac with fetal heart activity at 1 month after transfer. The implantation rate was calculated as the number of gestational sacs divided by the number of transferred embryos.

\section{Statistical Analysis}

Continuous variables were represented as means \pm standard deviations, and tested for normality using the Shapiro-Wilk and Kolmogorov-Smirnov tests. Data complying with the normal distribution were compared by Student's $t$-test or oneway analysis of variance, while those that did not were compared by Mann-Whitney $U$-test or Kruskal-Wallis test. For categorical variables, data were expressed as numbers and percentages of total, and compared by Pearson's Chi-square test or Fisher's exact test as appropriate.

Propensity score matching was performed to balance baseline characteristics between the vaccinated and unvaccinated groups at a ratio of 1:4. We selected 14 covariates to estimate propensity scores in a logistic regression model, including age, BMI, infertility duration, type of infertility, infertility diseases (6 variables), antral follicle count, previous IVF attempts, ovarian stimulation protocol, and fertilization method. The nearest-neighbor random matching algorithm was applied without replacement, and a caliper width of 0.05 standard deviations was specified. The success of matching was assessed using propensity score histograms. SAS version 9.4 (SAS Institute, USA) was used for all data analyses. All tests were two-tailed and $P<0.05$ was considered as statistically significant.

\section{Results}

A total of 2185 eligible women were included in the analysis. There were $150(6.9 \%)$ patients who received two dosages of inactivated SARS-CoV-2 vaccines, while the majority of patients $(\mathrm{n}=2035 ; 93.1 \%)$ were unvaccinated. For vaccinated women, the mean time interval between the second vaccine dose and IVF treatment start was $72.4 \pm 57.0$ days.

Baseline characteristics according to the vaccination status are depicted in Table 1. Before matching, the two groups differed significantly in maternal age $(P<0.001)$, tubal factor infertility $(P=0.030)$ and ovarian stimulation protocol $(P<0.001)$. After propensity score matching, 146 women remained in the vaccinated group and 584 remained in the unvaccinated group. All variables were similarly adjusted, and the propensity score distributions are presented in Figure 2.

Table 2 shows the cycle characteristics and laboratory outcomes grouped by the vaccination status. In the matched cohort, no significant differences were observed regarding stimulation duration, total gonadotropin dose, as well as serum sex hormone level, endometrial thickness and number of $\geq 14 \mathrm{~mm}$ follicles on trigger day. The number of retrieved oocytes, MII oocytes, 2PN oocytes, cleaved embryos, and good-quality day 3 embryos were also similar between the two groups. Consistently, there were no statistical significances when oocyte retrieval rate, mature oocyte rate, normal fertilization rate, cleavage rate, good-quality embryo rate, blastocyst formation rate, and available blastocyst rate were compared.

Pregnancy outcomes following fresh embryo transfer are displayed in Table 3. In the matched cohort, a total of 302 patients completed 66 transfer cycles in the vaccinated group and 236 cycles in the unvaccinated group. The number of embryos transferred and embryo stage did not differ significantly between groups. Clinical pregnancy rate per cycle was $59.1 \%$ and $63.6 \%$ in vaccinated and unvaccinated women, respectively $(P=0.507)$. Similarly, the two groups were comparable in the rates of biochemical pregnancy $(65.2 \%$ vs $73.7 \% ; P=0.171)$ and implantation $(45.4 \%$ vs $46.7 \% ; P=$ $0.803)$.

Based on the time interval from complete vaccination to cycle initiation, vaccinated patients were further subdivided into three categories: $\leq 1$ month, $>1-2$ months, and $>2$ months. As demonstrated in Table 4 , comparison among groups did not reveal any significant differences in either laboratory or pregnancy outcomes. 
Table I Baseline Characteristics Grouped by the Vaccination Status

\begin{tabular}{|c|c|c|c|c|c|c|}
\hline & \multicolumn{2}{|l|}{ Before PSM } & \multirow[t]{2}{*}{ P-value } & \multicolumn{2}{|l|}{ After PSM } & \multirow[t]{2}{*}{ P-value } \\
\hline & $\begin{array}{l}\text { Vaccinated } \\
(n=150)\end{array}$ & $\begin{array}{l}\text { Unvaccinated } \\
(n=2035)\end{array}$ & & $\begin{array}{l}\text { Vaccinated } \\
(n=146)\end{array}$ & $\begin{array}{l}\text { Unvaccinated } \\
(n=584)\end{array}$ & \\
\hline Age (years) & $33.7 \pm 5.6$ & $31.8 \pm 5.1$ & $<0.001$ & $33.6 \pm 5.5$ & $33.4 \pm 5.5$ & 0.781 \\
\hline Body mass index $\left(\mathrm{kg} / \mathrm{m}^{2}\right)$ & $22.1 \pm 3.1$ & $22.3 \pm 3.1$ & 0.582 & $22.1 \pm 3.1$ & $22.3 \pm 3.0$ & 0.493 \\
\hline Infertility duration (years) & $4.3 \pm 3.0$ & $4.3 \pm 3.2$ & 0.949 & $4.4 \pm 2.9$ & $4.3 \pm 3.3$ & 0.786 \\
\hline Type of infertility, n (\%) & & & 0.084 & & & 0.968 \\
\hline Primary & $45(30.0)$ & $754(37.1)$ & & $45(30.8)$ & $179(30.7)$ & \\
\hline Secondary & $105(70.0)$ & $|28|(62.9)$ & & $101(69.2)$ & $405(69.3)$ & \\
\hline \multicolumn{7}{|l|}{ Infertility diseases } \\
\hline Tubal factor, n (\%) & $88(58.7)$ & $1370(67.3)$ & 0.030 & $87(59.6)$ & $342(58.6)$ & 0.822 \\
\hline Male factor, n (\%) & $37(24.7)$ & $472(23.2)$ & 0.681 & $36(24.7)$ & $149(25.5)$ & 0.832 \\
\hline Ovulatory dysfunction, n (\%) & $22(14.7)$ & $280(13.8)$ & 0.756 & $21(14.4)$ & $88(15.1)$ & 0.836 \\
\hline Endometriosis, n (\%) & $15(10.0)$ & $142(7.0)$ & 0.167 & $14(9.6)$ & $54(9.2)$ & 0.899 \\
\hline Intrauterine adhesion, $\mathrm{n}(\%)$ & $22(14.7)$ & $223(11.0)$ & 0.165 & $21(14.4)$ & $85(14.6)$ & 0.958 \\
\hline Unexplained, n (\%) & $0(0)$ & $8(0.4)$ & 1.000 & $0(0)$ & $0(0)$ & 1.000 \\
\hline Antral follicle count & $11.7 \pm 7.0$ & $12.8 \pm 6.6$ & 0.071 & $11.7 \pm 6.8$ & $11.9 \pm 6.9$ & 0.764 \\
\hline Previous IVF attempts, n (\%) & & & 0.069 & & & 0.804 \\
\hline 0 & $112(74.7)$ & $15 I \mid(74.3)$ & & $109(74.7)$ & $421(72.1)$ & \\
\hline $\mathrm{I}-2$ & $32(21.3)$ & $345(17.0)$ & & $3 \mid(2 \mid .2)$ & $134(23.0)$ & \\
\hline$\geq 3$ & $6(4.0)$ & $179(8.8)$ & & $6(4.1)$ & $29(5.0)$ & \\
\hline $\begin{array}{l}\text { Ovarian stimulation protocol, } \\
\text { n (\%) }\end{array}$ & & & $<0.001$ & & & 0.854 \\
\hline GnRH agonist & $86(57.3)$ & $1550(76.2)$ & & $86(58.9)$ & $329(56.3)$ & \\
\hline $\mathrm{GnRH}$ antagonist & $33(22.0)$ & $197(9.7)$ & & $30(20.5)$ & $128(21.9)$ & \\
\hline Others & 31 (20.7) & $288(14.2)$ & & $30(20.5)$ & $127(2 \mid .7)$ & \\
\hline Fertilization method, $\mathrm{n}(\%)^{*}$ & & & 0.095 & & & 0.826 \\
\hline IVF & $100(67.1)$ & $1348(67.1)$ & & $100(69.0)$ & $386(66.8)$ & \\
\hline ICSI & $46(30.9)$ & $539(26.8)$ & & $42(29.0)$ & $176(30.4)$ & \\
\hline IVF+ICSI & $3(2.0)$ & $|2|(6.0)$ & & $3(2.1)$ & $16(2.8)$ & \\
\hline
\end{tabular}

Notes: Data are presented as mean \pm standard deviation or number (percentage). *One vaccinated woman and 27 unvaccinated women had no available oocytes for fertilization.

Abbreviations: PSM, propensity score matching; IVF, in vitro fertilization; ICSI, intracytoplasmic sperm injection; GnRH, gonadotropin-releasing hormone.

\section{Discussion}

The results of our retrospective cohort study demonstrated that inactivated SARS-CoV-2 vaccination in females had no negative influence on IVF treatment. Furthermore, both laboratory and pregnancy outcomes were not significantly altered by different time intervals from vaccination to cycle initiation.

Response to ovarian stimulation with gonadotropins is considered to be the most reliable sign of decreased ovarian reserve. ${ }^{20}$ In the present study, comparable responses were found between vaccinated and unvaccinated women, implying no deleterious impact of inactivated COVID-19 vaccine on ovarian reserve/oocytes pool. Consistently, a recent prospective study also demonstrated that ovarian reserve as assessed by circulating anti-Müllerian hormone concentrations did not change significantly before and three months after two mRNA SARS-CoV-2 vaccinations (5.30 \pm 4.29 vs 5.30 $\pm 4.50 \mu \mathrm{g} / \mathrm{L} ; P=0.11) .{ }^{21}$ Furthermore, no association was observed between anti-SARS-CoV-2 antibody levels and AMH levels after controlling for age, suggesting that the potential negative effect of immune response following vaccination on fertility may be limited. Similar concern was raised when human papillomavirus (HPV) vaccine was introduced, especially as it was recommended for adolescents and young women. Nonetheless, this was also refuted by a populationbased cohort study of 199,078 patients showing no elevated risk of premature ovarian insufficiency after HPV vaccination. $^{22}$ 

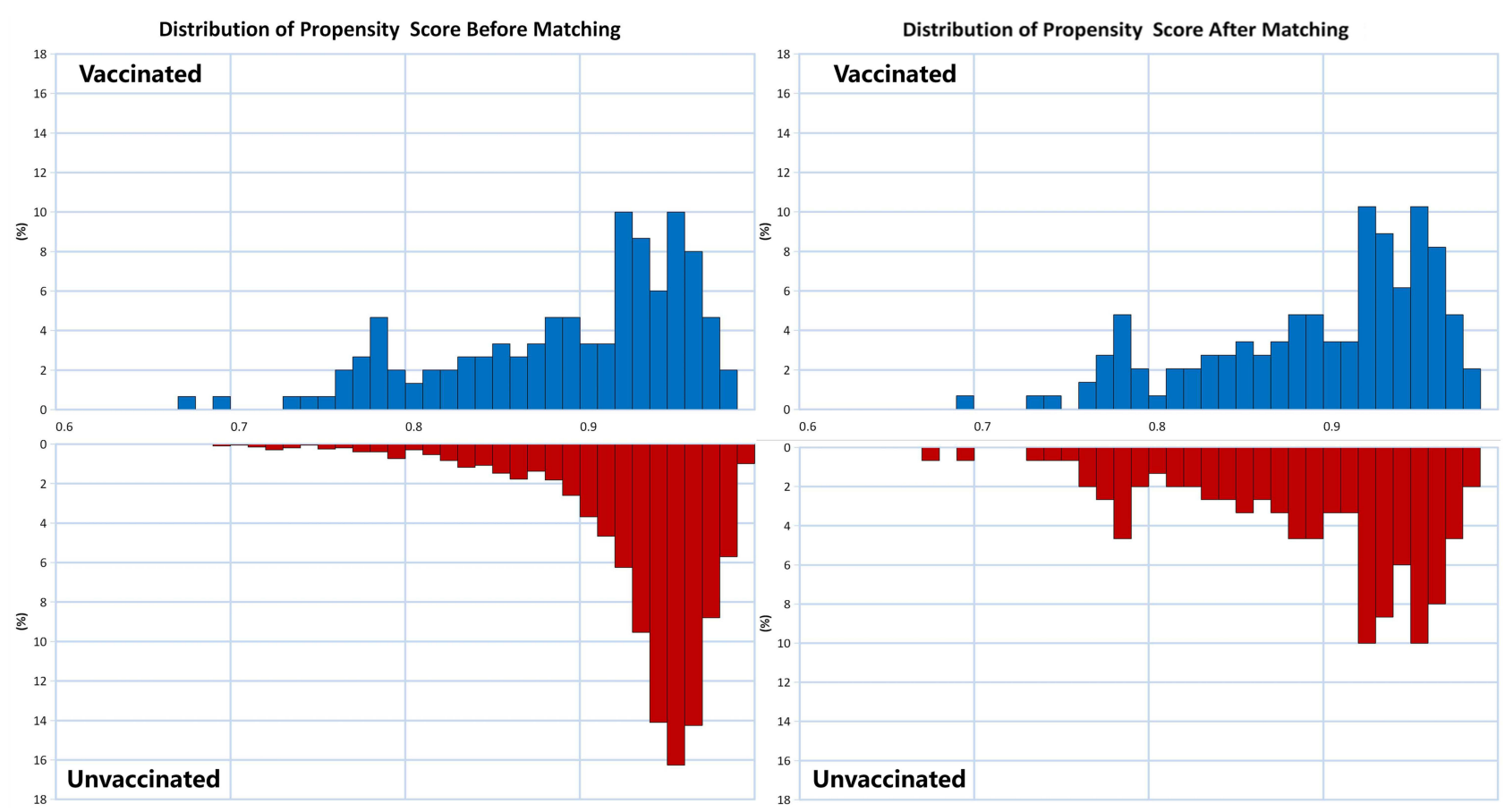

Figure 2 Propensity score histograms of vaccinated and unvaccinated women before and after matching. The propensity scores were successfully balanced between the two groups after matching.

To our knowledge, only two prior studies have tried to investigate the impact of BNT162b2 mRNA COVID-19 vaccine on IVF outcomes. The first study by Orvieto et $\mathrm{al}^{15}$ included 36 women undergoing consecutive IVF cycles, and observed similar ovarian stimulation and embryological characteristics before and after vaccination. Additionally, three pregnancies were recorded out of 10 patients who received embryo transfer, implying an acceptable pregnancy rate (30\%) following vaccination. In another cohort study by Bentov et al, ${ }^{14}$ nine vaccinated women were compared with 14 non-vaccinated controls. No significant differences were found in any surrogate parameters for ovarian steroidogenesis (trigger day estradiol and progesterone level in serum and follicular fluid [FF]), response to the luteinizing hormone/hCG trigger (oocyte retrieval rate, mature oocyte rate, and ratio of oocytes retrieved to oocyte retrieval day serum progesterone level), as well as oocyte quality biomarkers (FF concentration of heparan sulfate proteoglycan 2). The current study, with a much larger sample size and a longer follow-up period, provides another observational evidence that inactivated SARSCoV-2 vaccine similarly does not affect patients' performance in IVF cycles.

SARS-CoV-2 has been suspected to be detrimental to female fertility due to the expression of angiotensin-converting enzyme 2 (ACE-2) and type 2 transmembrane serine protease (TMPRSS-2) receptors in the reproductive system. ${ }^{23}$ However, most clinical studies subsequently showed that the ovarian function and IVF outcomes did not obviously change in patients with a history of infection. ${ }^{14,24-26}$ Regarding the COVID-19 vaccine, immunity-related effects have been suggested to be the biological mechanisms linked with potential infertility. On the one hand, immune cells are involved in the intra-ovarian processes and release of cytokines required for normal follicular development. ${ }^{27,28}$ In previous studies, women with increased $\mathrm{T}$ cell activation were reported to have a greater likelihood of experiencing embryo implantation failure. ${ }^{29,30}$ Indeed, vaccine administration could elicit high SARS-CoV-2 neutralizing antibody production alongside antigen-specific cluster of differentiation 8 (CD8)+ and T-helper 1 (Th1) type CD4+ T cell response. ${ }^{31}$ Local involvement of the ovary has also been reflected by the presence of anti-SARS-CoV-2 immunoglobulin $\mathrm{G}$ in the FF of vaccinated versus non-vaccinated IVF patients. ${ }^{14}$ Therefore, the activated immune response after vaccination may lead to unsuccessful IVF treatment. On the other hand, vaccination might induce the synthesis of antiphospholipid antibodies (aPLs), such as anti-cardiolipin, anti-b2 glycoprotein I and anti-phosphatidylserine/prothrombin, and even promote the development of immune thrombosis and thrombocytopenia. ${ }^{32-34}$ The presence of 
Table 2 Cycle Characteristics and Laboratory Outcomes Grouped by the Vaccination Status

\begin{tabular}{|c|c|c|c|c|c|c|}
\hline & \multicolumn{2}{|l|}{ Before PSM } & \multirow[t]{2}{*}{ P-value } & \multicolumn{2}{|l|}{ After PSM } & \multirow[t]{2}{*}{ P-value } \\
\hline & Vaccinated $(n=150)$ & Unvaccinated $(n=2035)$ & & Vaccinated $(n=146)$ & Unvaccinated $(n=584)$ & \\
\hline Stimulation duration (days) & $10.2 \pm 2.3$ & $10.4 \pm 2.7$ & 0.452 & $10.3 \pm 2.3$ & $9.9 \pm 2.8$ & 0.084 \\
\hline Total gonadotropin dose (IU) & $2012.3 \pm 722.7$ & $2011.9 \pm 829.3$ & 0.995 & $2019.6 \pm 721.2$ & $|969.1 \pm 8| 2.4$ & 0.493 \\
\hline LH level on trigger day $(\mathrm{mlU} / \mathrm{mL})$ & $2.4 \pm 1.6$ & $2.3 \pm 3.1$ & 0.390 & $2.4 \pm 1.6$ & $2.7 \pm 3.9$ & 0.234 \\
\hline $\mathrm{E}_{2}$ level on trigger day $(\mathrm{pg} / \mathrm{mL})$ & $1764.5 \pm 15 \mid 3.0$ & $1920.1 \pm \mid 483.2$ & 0.220 & $1770.2 \pm 1525.3$ & $1698.1 \pm 1344.6$ & 0.606 \\
\hline $\mathrm{P}$ level on trigger day $(\mathrm{ng} / \mathrm{mL})$ & $0.3 \pm 0.3$ & $0.4 \pm 0.4$ & 0.226 & $0.3 \pm 0.3$ & $0.3 \pm 0.3$ & 0.873 \\
\hline EMT on trigger day $(\mathrm{mm})$ & $9.9 \pm 3.1$ & $10.4 \pm 2.8$ & 0.068 & $10.0 \pm 3.1$ & $10.1 \pm 2.9$ & 0.761 \\
\hline No. of $\geq 14 \mathrm{~mm}$ follicles on trigger day & $7.4 \pm 4.4$ & $8.2 \pm 4.3$ & 0.019 & $7.4 \pm 4.4$ & $7.6 \pm 4.4$ & 0.730 \\
\hline No. of oocytes retrieved & $9.9 \pm 7.2$ & $11.1 \pm 6.9$ & 0.049 & $9.9 \pm 7.1$ & $9.9 \pm 6.7$ & 0.893 \\
\hline No. of MII oocytes & $8.2 \pm 6.1$ & $8.8 \pm 5.7$ & 0.238 & $8.3 \pm 6.1$ & $7.9 \pm 5.6$ & 0.536 \\
\hline No. of $2 \mathrm{PN}$ oocytes & $6.3 \pm 4.9$ & $6.8 \pm 4.7$ & 0.253 & $6.4 \pm 4.9$ & $6.2 \pm 4.7$ & 0.746 \\
\hline No. of cleaved embryos & $6.1 \pm 4.8$ & $6.5 \pm 4.6$ & 0.335 & $6.2 \pm 4.8$ & $6.0 \pm 4.5$ & 0.686 \\
\hline No. of good-quality embryos on day 3 & $1.9 \pm 2.1$ & $1.8 \pm 2.0$ & 0.434 & $2.0 \pm 2.2$ & $1.7 \pm 2.0$ & 0.144 \\
\hline Oocyte retrieval rate (\%) & $132.8 \pm 43.9$ & $136.2 \pm 53.5$ & 0.362 & $132.0 \pm 43.8$ & $132.6 \pm 54 . \mid$ & 0.888 \\
\hline Mature oocyte rate (\%) & $83.2 \pm 17.6$ & $81.4 \pm 28.5$ & 0.264 & $83.6 \pm 17.5$ & $82.4 \pm 19.3$ & 0.503 \\
\hline Normal fertilization rate (\%) & $71.2 \pm 23.5$ & $67.5 \pm 23.3$ & 0.064 & $71.1 \pm 23.3$ & $70.2 \pm 23.9$ & 0.681 \\
\hline Cleavage rate $(\%)$ & $97.8 \pm 6.3$ & $96.2 \pm 10.7$ & 0.005 & $97.9 \pm 6.2$ & $96.7 \pm 11.1$ & 0.088 \\
\hline Good-quality embryo rate (\%) & $34.0 \pm 30.6$ & $28.6 \pm 27.0$ & 0.039 & $33.5 \pm 29.8$ & $29.9 \pm 28.6$ & 0.184 \\
\hline Blastocyst formation rate (\%) & $74.5 \pm 30.6$ & $71.2 \pm 32.1$ & 0.308 & $74.5 \pm 30.5$ & $71.6 \pm 31.3$ & 0.407 \\
\hline Available blastocyst rate (\%) & $71.8 \pm 28.6$ & $72 \pm 30.0$ & 0.942 & $72.2 \pm 27.9$ & $71.3 \pm 30.2$ & 0.803 \\
\hline
\end{tabular}

Note: Data are presented as mean \pm standard deviation.

Abbreviations: PSM, propensity score matching; LH, luteinizing hormone; $E_{2}$, estradiol; P, progesterone; EMT, endometrial thickness; MII, metaphase II; 2PN, two pronuclei. 
Table 3 Pregnancy Outcomes Grouped by the Vaccination Status

\begin{tabular}{|c|c|c|c|c|c|c|}
\hline & \multicolumn{2}{|l|}{ Before PSM } & \multirow[t]{2}{*}{ P-value } & \multicolumn{2}{|l|}{ After PSM } & \multirow[t]{2}{*}{ P-value } \\
\hline & $\begin{array}{l}\text { Vaccinated } \\
(n=66)\end{array}$ & $\begin{array}{l}\text { Unvaccinated } \\
(n=I 103)\end{array}$ & & $\begin{array}{l}\text { Vaccinated } \\
(n=66)\end{array}$ & $\begin{array}{l}\text { Unvaccinated } \\
(n=236)\end{array}$ & \\
\hline No. of embryos transferred, n (\%) & & & 0.126 & & & 0.093 \\
\hline Single & $24(36.4)$ & $305(27.7)$ & & $24(36.4)$ & $61(25.8)$ & \\
\hline Double & $42(63.6)$ & $798(72.3)$ & & $42(63.6)$ & $175(74.2)$ & \\
\hline Embryo stage at transfer, $\mathrm{n}(\%)$ & & & $0.24 I$ & & & 0.141 \\
\hline Cleavage & $46(69.7)$ & $839(76.1)$ & & $46(69.7)$ & $185(78.4)$ & \\
\hline Blastocyst & $20(30.3)$ & $264(23.9)$ & & $20(30.3)$ & $5 \mathrm{I}(2 \mathrm{I} .6)$ & \\
\hline Embryo transfer strategy, n (\%) & & & 0.101 & & & 0.060 \\
\hline Double cleavage & $40(60.6)$ & 787 (7I.4) & & $40(60.6)$ & $174(73.7)$ & \\
\hline Single blastocyst & $18(27.3)$ & $253(22.9)$ & & $18(27.3)$ & $50(21.2)$ & \\
\hline Single cleavage & $6(9.1)$ & $52(4.7)$ & & $6(9.1)$ & II (4.7) & \\
\hline Double blastocyst & $2(3.0)$ & II (I.0) & & $2(3.0)$ & $\mathrm{I}(0.4)$ & \\
\hline $\begin{array}{l}\text { Biochemical pregnancy rate, } n / N \\
\text { (\%) }\end{array}$ & $43 / 66(65.2)$ & $822 / 1103(74.5)$ & 0.092 & $43 / 66(65.2)$ & $174(73.7)$ & 0.171 \\
\hline Clinical pregnancy rate, $\mathrm{n} / \mathrm{N}$ (\%) & $39 / 66(59.1)$ & $7 / 2 / 1103(64.6)$ & 0.369 & $39 / 66(59.1)$ & $150(63.6)$ & 0.507 \\
\hline Implantation rate, $\mathrm{n} / \mathrm{N}(\%)$ & $49 / 108(45.4)$ & $922 / 1901(48.5)$ & 0.527 & $49 / 108(45.4)$ & $|92 / 4| \mid(46.7)$ & 0.803 \\
\hline
\end{tabular}

Note: Data are presented as number (percentage).

Abbreviation: PSM, propensity score matching.

aPLs may consequently lead to infertility, implantation failure as well as recurrent pregnancy loss. ${ }^{35}$ Though seemingly rational, these hypotheses again did not match directly with clinical data from our and other studies, ${ }^{14,15}$ suggesting that the immunity-medicated effects following vaccination could be limited to female pregnancy.

Timing of IVF start after the completion of vaccination has been inconsistent among different society guidelines. According to the Expert Group for Beijing Human Assisted Reproductive Technology Center for Quality Control and Improvement, a general postponement of 1 month is recommended. ${ }^{36}$ Considering the insufficient information on the impact of COVID-19 vaccine on gametes, embryo implantation and early pregnancy, and to allow time for antibody development, the European Society of Human Reproduction and Embryology advised women to wait for up to 2 months in a more cautious approach. ${ }^{37}$ Instead, the American Society for Reproductive Medicine Coronavirus/COVID-19 Task Force recommended that patients should be vaccinated at the soonest possible time, but those scheduled for oocyte retrieval or embryo transfer should be avoided for at least 3 days prior and 3 days after the procedure, given the time required for recovery from common side effects of vaccines (eg, fever, chills, fatigue, myalgia and headaches). ${ }^{38}$ In practice, Orvieto et $\mathrm{l}^{15}$ reported the time interval ranged between 7 and 85 days, while some patients received their first dose during ovarian stimulation (only 8 days prior to oocyte retrieval) in another study. ${ }^{14}$ Consistent with the Beijing guideline, ${ }^{36}$ the majority of our patients resumed IVF cycles after 1 month, while the laboratory and pregnancy outcomes were comparable to those within 1 month. This reassuring finding should provide valuable guidance for vaccinated women who wish to proceed treatment at their earliest convenience, although larger cohort studies are needed for further confirmation.

To date, this is the first study to examine the impact of inactivated SARS-CoV-2 vaccination on IVF outcomes. Vaccine administration information was ascertained via immunization records that can be accessed in various mobile apps, thus minimizing the possibility of recall bias by patients. We also used a matched cohort of propensity scorebalanced women to control for potential confounders. Although propensity score matching is no substitute for randomization, it remains one of the best methods for causal inference from observational data. ${ }^{39}$

Some limitations should be acknowledged in the current work. First, it was a retrospective cohort study with inherent bias, and some confounding factors may be missed. For example, the vaccination status in males was not included for adjustment. While mRNA SARS-CoV-2 vaccine does not pose a detrimental influence on semen parameters, ${ }^{40}$ whether this conclusion applies to inactivated vaccines deserves further investigation. Also, the characteristics of vaccinated 
Table 4 Cycle Outcomes Based on Time Interval from Complete Vaccination to Cycle Initiation

\begin{tabular}{|c|c|c|c|c|}
\hline & $\leq$ I Month & >I-2 Months & $>2$ Months & $P$-value \\
\hline Laboratory Outcomes & $\mathrm{n}=37$ & $\mathrm{n}=42$ & $n=71$ & \\
\hline No. of oocytes retrieved & $9.6 \pm 7.8$ & $10.8 \pm 7.4$ & $9.6 \pm 6.7$ & 0.668 \\
\hline Mature oocyte rate (\%) & $80.6 \pm 18.6$ & $86.1 \pm 13.7$ & $82.8 \pm 19.1$ & 0.374 \\
\hline Normal fertilization rate (\%) & $71.2 \pm 24.4$ & $75.3 \pm 19.3$ & $68.8 \pm 25.3$ & 0.370 \\
\hline Cleavage rate $(\%)$ & $98.2 \pm 5.7$ & $98.1 \pm 5.2$ & $97.4 \pm 7.2$ & 0.797 \\
\hline Good-quality embryo rate (\%) & $29.8 \pm 29.5$ & $32.3 \pm 29.5$ & $37.2 \pm 31.8$ & 0.457 \\
\hline Blastocyst formation rate (\%) & $72.2 \pm 29.7$ & $75.7 \pm 29.7$ & $74.9 \pm 32.3$ & 0.906 \\
\hline Available blastocyst rate (\%) & $67.3 \pm 33.9$ & $71.6 \pm 23.5$ & $74.3 \pm 29.1$ & 0.653 \\
\hline Pregnancy outcomes & $\mathrm{n}=12$ & $\mathrm{n}=18$ & $\mathrm{n}=36$ & \\
\hline Biochemical pregnancy rate, $\mathrm{n} / \mathrm{N}$ (\%) & $9 / 12(75.0)$ & $9 / 18(50.0)$ & $25 / 36(69.4)$ & 0.269 \\
\hline Clinical pregnancy rate, $\mathrm{n} / \mathrm{N}(\%)$ & $9 / 12(75.0)$ & $8 / 18(44.4)$ & $22 / 36(61.1)$ & 0.233 \\
\hline Implantation rate, $\mathrm{n} / \mathrm{N}(\%)$ & $12 / 20(60.0)$ & $9 / 28(32.1)$ & $28 / 60(46.7)$ & 0.154 \\
\hline
\end{tabular}

Note: Data are presented as mean \pm standard deviation or number (percentage).

patients were collected after vaccination, and further comparisons should be made with those before vaccination, such as menstrual cycle, sex hormone level and ovulatory function. Second, although our study thus far was the largest compared with other studies, ${ }^{14,15}$ the sample size of 146 in the vaccinated group was still small. The limited statistical power, together with the single-center setting, may thus affect the robustness and generalizability of our conclusion. Third, data on live birth, the primary outcome of IVF treatment, is lacking owing to the follow-up period. For safety reasons, continued monitoring is needed to further evaluate obstetrical, perinatal and childhood outcomes associated with maternal COVID-19 vaccination. Finally, since the study was conducted in Chinese women with inactivated SARSCoV-2 vaccination, the application of our results should not be directly extrapolated to other ethnic/racial populations or vaccine types. In addition, only fresh IVF cycles were analyzed, while the finding remains to be validated in patients undergoing frozen-thawed embryo transfer, intrauterine insemination or other assisted reproductive treatment.

\section{Conclusions}

In summary, our study provided the first-time evidence that inactivated SARS-CoV-2 vaccination in females did not result in any measurable effects on IVF treatment, including cycle characteristics, laboratory parameters and pregnancy outcomes. Given the low vaccination coverage rate, this finding should be reassuring and useful to aid both fertility specialists and patients in counselling and decision-making process. Further prospective studies with larger cohort size and longer follow-up are warranted to validate our conclusion.

\section{Data Sharing Statement}

The datasets used and/or analyzed during the current study are available from the corresponding author on reasonable request (Qiongfang Wu; E-mail: wuqfivf@126.com).

\section{Ethics Approval and Informed Consent}

The study was approved by the Ethics Committee of Jiangxi Maternal and Child Health Hospital (No. 2021-02) and was conducted in accordance with the Declaration of Helsinki. Informed contents were obtained from patients for data collection with scientific use.

\section{Acknowledgments}

The authors would like to thank all working staff at the Center for Reproductive Medicine of JMCHH for their active involvement and collaboration in project administration. Moreover, the authors are sincerely grateful for the infertile couples who participated in this study. 


\section{Funding}

This study was funded by the National Natural Science Foundation of China (81960288) and the Key Research and Development Program of Jiangxi Province (20203BBGL73159).

\section{Disclosure}

The authors report no conflicts of interest in this work.

\section{References}

1. WHO. WHO coronavirus disease (COVID-19) dashboard. Available from: https://covid19.who.int/. Accessed October 3, 2021.

2. Allotey J, Stallings E, Bonet M, et al. Clinical manifestations, risk factors, and maternal and perinatal outcomes of coronavirus disease 2019 in pregnancy: living systematic review and meta-analysis. BMJ. 2020;370:m3320. doi:10.1136/bmj.m3320

3. Chmielewska B, Barratt I, Townsend R, et al. Effects of the COVID-19 pandemic on maternal and perinatal outcomes: a systematic review and meta-analysis. Lancet Glob Health. 2021;9(6):e759-e772.

4. Wei SQ, Bilodeau-Bertrand M, Liu S, Auger N. The impact of COVID-19 on pregnancy outcomes: a systematic review and meta-analysis. CMAJ. 2021;193(16):E540-E548. doi:10.1503/cmaj.202604

5. National Health Commission of the People's Republic of China. COVID-19 vaccination status. Available from: http://www.nhc.gov.cn/. Accessed October 3, 2021.

6. Callaway E. The race for coronavirus vaccines: a graphical guide. Nature. 2020;580(7805):576-577. doi:10.1038/d41586-020-01221-y

7. Al Kaabi N, Zhang Y, Xia S, et al. Effect of 2 inactivated SARS-CoV-2 vaccines on symptomatic COVID-19 infection in adults: a randomized clinical trial. JAMA. 2021;326(1):35-45. doi:10.1001/jama.2021.8565

8. Sadoff J, Gray G, Vandebosch A, et al. Safety and efficacy of single-dose Ad26.COV2.S vaccine against Covid-19. N Engl J Med. 2021;384 (23):2187-2201. doi:10.1056/NEJMoa2101544

9. Thomas SJ, Moreira ED Jr, Kitchin N, et al. Safety and efficacy of the BNT162b2 mRNA Covid-19 vaccine through 6 months. $N$ Engl J Med. 2021;385(19):1761-1773. doi:10.1056/NEJMoa2110345

10. Blake Evans M, Alexander C, Barnard E, et al. COVID-19 vaccine and infertility: baseless claims and unfounded social media panic. ASRM, Fertility and Sterility; 2021. Available from: https://www.fertstertdialog.com/posts/covid-19-vaccine-and-infertility-baseless-claims-and-unfounded -social-media-panic. Accessed January 26, 2022.

11. Iacobucci G. Covid-19: no evidence that vaccines can affect fertility, says new guidance. BMJ. 2021;372:n509. doi:10.1136/bmj.n509

12. Flynn AC, Kavanagh K, Smith AD, Poston L, White SL. The impact of the COVID-19 pandemic on pregnancy planning behaviors. Women's Health Rep. 2021;2(1):71-77.

13. Skjefte M, Ngirbabul M, Akeju O, et al. COVID-19 vaccine acceptance among pregnant women and mothers of young children: results of a survey in 16 countries. Eur J Epidemiol. 2021;36(2):197-211. doi:10.1007/s10654-021-00728-6

14. Bentov Y, Beharier O, Moav-Zafrir A, et al. Ovarian follicular function is not altered by SARS-CoV-2 infection or BNT162b2 mRNA COVID-19 vaccination. Hum Reprod. 2021;36(9):2506-2513. doi:10.1093/humrep/deab182

15. Orvieto R, Noach-Hirsh M, Segev-Zahav A, Haas J, Nahum R, Aizer A. Does mRNA SARS-CoV-2 vaccine influence patients' performance during IVF-ET cycle? Reprod Biol Endocrinol. 2021;19(1):69. doi:10.1186/s12958-021-00757-6

16. Zhu FC, Guan XH, Li YH, et al. Immunogenicity and safety of a recombinant adenovirus type-5-vectored COVID-19 vaccine in healthy adults aged 18 years or older: a randomised, double-blind, placebo-controlled, Phase 2 trial. Lancet. 2020;396(10249):479-488. doi:10.1016/S01406736(20)31605-6

17. Yang S, Li Y, Dai L, et al. Safety and immunogenicity of a recombinant tandem-repeat dimeric RBD-based protein subunit vaccine (ZF2001) against COVID-19 in adults: two randomised, double-blind, placebo-controlled, Phase 1 and 2 trials. Lancet Infect Dis. 2021;21(8):1107-1119. doi:10.1016/S1473-3099(21)00127-4

18. Cummins JM, Breen TM, Harrison KL, Shaw JM, Wilson LM, Hennessey JF. A formula for scoring human embryo growth rates in in vitro fertilization: its value in predicting pregnancy and in comparison with visual estimates of embryo quality. $J$ Vitro Fert Embryo Transfer. 1986;3 (5):284-295. doi:10.1007/BF01133388

19. Gardner DK, Schoolcraft WB. In vitro culture of human blastocyst. In: Jansen R, Mortimer D, editors. Towards Reproductive Certainty: Infertility and Genetics Beyond 1999. Carnforth: Parthenon Press; 1999:378-388.

20. Gleicher N, Barad D. Unexplained infertility: does it really exist? Hum Reprod. 2006;21(8):1951-1955. doi:10.1093/humrep/del135

21. Mohr-Sasson A, Haas J, Abuhasira S, et al. The effect of Covid-19 mRNA vaccine on serum anti-Müllerian hormone levels. Hum Reprod. 2021. doi:10.1093/humrep/deab282

22. Naleway AL, Mittendorf KF, Irving SA, et al. Primary ovarian insufficiency and adolescent vaccination. Pediatrics. 2018;142(3):e20180943. doi:10.1542/peds.2018-0943

23. Chandi A, Jain N. State of ART in the COVID-19 era and consequences on human reproductive system. Biol Reprod. 2021;105(4):808-821. doi:10.1093/biolre/ioab122

24. Li K, Chen G, Hou H, et al. Analysis of sex hormones and menstruation in COVID-19 women of child-bearing age. Reprod Biomed Online. 2021;42(1):260-267. doi:10.1016/j.rbmo.2020.09.020

25. Orvieto R, Segev-Zahav A, Aizer A. Does COVID-19 infection influence patients' performance during IVF-ET cycle?: an observational study. Gynecol Endocrinol. 2021;37(10):895-897. doi:10.1080/09513590.2021.1918080

26. Wang M, Yang Q, Ren X, et al. Investigating the impact of asymptomatic or mild SARS-CoV-2 infection on female fertility and in vitro fertilization outcomes: a retrospective cohort study. EClinicalMedicine. 2021;38:101013. doi:10.1016/j.eclinm.2021.101013

27. Kollmann Z, Schneider S, Fux M, Bersinger NA, von Wolff M. Gonadotrophin stimulation in IVF alters the immune cell profile in follicular fluid and the cytokine concentrations in follicular fluid and serum. Hum Reprod. 2017;32(4):820-831. doi:10.1093/humrep/dex005 
28. Lachapelle MH, Hemmings R, Roy DC, Falcone T, Miron P. Flow cytometric evaluation of leukocyte subpopulations in the follicular fluids of infertile patients. Fertil Steril. 1996;65(6):1135-1140. doi:10.1016/S0015-0282(16)58327-7

29. Gallinelli A, Roncaglia R, Matteo ML, Ciaccio I, Volpe A, Facchinetti F. Immunological changes and stress are associated with different implantation rates in patients undergoing in vitro fertilization-embryo transfer. Fertil Steril. 2001;76(1):85-91. doi:10.1016/S0015-0282(01) 01826-X

30. Yang KM, Ntrivalas E, Cho HJ, et al. Women with multiple implantation failures and recurrent pregnancy losses have increased peripheral blood T cell activation. Am J Reprod Immunol. 2010;63(5):370-378. doi:10.1111/j.1600-0897.2010.00811.x

31. Soleimanpour S, Yaghoubi A. COVID-19 vaccine: where are we now and where should we go? Expert Rev Vaccines. 2021;20(1):23-44. doi:10.1080/14760584.2021.1875824

32. Cruz-Tapias P, Blank M, Anaya JM, Shoenfeld Y. Infections and vaccines in the etiology of antiphospholipid syndrome. Curr Opin Rheumatol. 2012;24(4):389-393. doi:10.1097/BOR.0b013e32835448b8

33. Sharifian-Dorche M, Bahmanyar M, Sharifian-Dorche A, Mohammadi P, Nomovi M, Mowla A. Vaccine-induced immune thrombotic thrombocytopenia and cerebral venous sinus thrombosis post COVID-19 vaccination; a systematic review. $J$ Neurol Sci. 2021;428:117607. doi:10.1016/j. jns.2021.117607

34. Liu T, Dai J, Yang Z, et al. Inactivated SARS-CoV-2 vaccine does not influence the profile of prothrombotic antibody nor increase the risk of thrombosis in a prospective Chinese cohort. Sci Bull. 2021;66(22):2312-2319. doi:10.1016/j.scib.2021.07.033

35. Backos M, Rai R, Regan L. Antiphospholipid antibodies and infertility. Hum Fertil. 2002;5(1):30-34. doi:10.1080/1464727992000199731

36. Expert Group for Beijing Human Assisted Reproductive Technology Center For Quality Control and Improvement. COVID-19 vaccination strategy for planning pregnancy and assisted reproductive technology treatment: expert recommendations [In Chinese]. Chin J Reprod Contracep. 2021;41 (4):296-299.

37. ESHRE COVID-19 Working Group. ESHRE STATEMENT ON COVID-19 vaccination and medically assisted reproduction. Available from: https://www.eshre.eu/Europe/Position-statements/COVID19/vaccination. Accessed October 3, 2021.

38. Coronavirus/COVID-19 Task Force of the American Society for Reproductive Medicine. ASRM patient management and clinical recommendations during the (coronavirus COVID-19)pandemic: update no. 13-variants, vaccines, and vaccination; February 22, 2021. Available from: https:// www.asrm.org/globalassets/asrm/asrm-content/news-and-publications/covid-19/covidtaskforceupdate13.pdf. Accessed October 3, 2021.

39. Austin PC. An introduction to propensity score methods for reducing the effects of confounding in observational studies. Multivariate Behav Res. 2011;46(3):399-424. doi:10.1080/00273171.2011.568786

40. Lifshitz D, Haas J, Lebovitz O, Raviv G, Orvieto R, Aizer A. Does mRNA SARS-CoV-2 vaccine detrimentally affect male fertility, as reflected by semen analysis? Reprod Biomed Online. 2021. doi:10.1016/j.rbmo.2021.09.021

Journal of Inflammation Research

\section{Publish your work in this journal}

The Journal of Inflammation Research is an international, peer-reviewed open-access journal that welcomes laboratory and clinical findings on the molecular basis, cell biology and pharmacology of inflammation including original research, reviews, symposium reports, hypothesis formation and commentaries on: acute/chronic inflammation; mediators of inflammation; cellular processes; molecular mechanisms; pharmacology and novel anti-inflammatory drugs; clinical conditions involving inflammation. The manuscript management system is completely online and includes a very quick and fair peer-review system. Visit http://www.dovepress.com/testimonials.php to read real quotes from published authors.

Submit your manuscript here: https://www.dovepress.com/journal-of-inflammation-research-journal 